EPJ Web of Conferences 116, 04008 (2016)

DOI: $10.1051 /$ epjconf/201611604008

(C) Owned by the authors, published by EDP Sciences, 2016

\title{
Predicted sensitivity of the KM3NeT/ARCA detector to a diffuse flux of cosmic neutrinos
}

\author{
R. Coniglione ${ }^{1}$, L.A. Fusco ${ }^{2,3, a}$, and D. Stransky ${ }^{4}$ for the KM3NeT Collaboration \\ ${ }^{1}$ INFN - Laboratori Nazionali del Sud, via S. Sofia 62, Catania 95123, Italy \\ ${ }^{2}$ Dipartimento di Fisica e Astronomia dell'Università, Viale Berti-Pichat 6/2, Bologna 40127, Italy \\ ${ }^{3}$ INFN - Sezione di Bologna, Viale Berti-Pichat 6/2, Bologna 40127, Italy \\ ${ }^{4}$ Friedrich-Alexander-Universität Erlangen-Nürnberg, Erlangen Centre for Astroparticle Physics, \\ Erwin-Rommel-Strasse 1, Erlangen 91058, Germany
}

\begin{abstract}
The KM3NeT Collaboration has started the construction of a research infrastructure hosting a network of underwater neutrino detectors in the Mediterranean Sea. Two instruments based on the same technology are being built: KM3NeT/ORCA to measure the neutrino mass hierarchy and to study atmospheric neutrino oscillations and KM3NeT/ARCA to detect high-energy cosmic neutrinos both in diffuse and point source mode. The excellent angular resolution of the ARCA detector, with an instrumented volume of about one Gton, will allow for an unprecedented exploration of the neutrino sky searching for neutrinos coming from defined sources of sky regions, like the Galactic Plane and the Fermi Bubbles. It will also look for diffuse high energy neutrino fluxes following the indication provided by the IceCube signal.

This contribution will report on the sensitivity of the KM3NeT/ARCA telescope with particular attention to the region of the Galactic Plane. Comparisons with theoretical expectations are also discussed.
\end{abstract}

\section{Introduction}

The KM3NeT Collaboration has started the construction of the next generation neutrino detectors, being built in the Mediterranean Sea. A large volume neutrino telescope, called ARCA [1], will be devoted to the search for high energy cosmic neutrinos while a more dense and compact neutrino detector, ORCA [1], will study the neutrino mass hierarchy looking at low energy atmospheric neutrino oscillations. Cherenkov light emitted by charged particles produced by all flavour neutrino interaction will be detected to reconstruct the neutrino direction and energy.

Both detectors will be built following a modular approach. The basic detector element is the detection unit, a vertical slender string kept taut by a top buoy and holding 18 pressure resistant glass spheres, each housing 313 -inch photomultipliers. 115 DUs will constitute a building block. ARCA will be made of two building blocks with an average spacing between DUs of $90 \mathrm{~m}$ and a vertical distance

\footnotetext{
a e-mail: lfusco@bo.infn.it
}

This is an Open Access article distributed under the terms of the Creative Commons Attribution License 4.0, which permits unrestricted use, distribution, and reproduction in any medium, provided the original work is properly cited. 
between DOMs on each DU of $36 \mathrm{~m}$; the total instrumented volume will be of the order of $1 \mathrm{~km}^{3}$. ORCA will comprise one building block having its DUs spaced horizontally by $20 \mathrm{~m}$ and an inter-DOM distance of $9 \mathrm{~m}$ for an instrumented volume of about 6 Mton.

The IceCube collaboration has observed cosmic neutrinos in various analyses [2-4], reporting a large excess of high energy neutrinos over the atmospheric flux expectations. The observed flux is compatible with isotropic origin and equipartition in the three neutrino flavours as expected from neutrino oscillations in vacuum [5]. Individual sources of this excess could not be identified, nor has the production mechanism for these high energy neutrinos been determined. Here, the one-flavour cosmic signal flux is parametrised as:

$$
E_{v}^{2} \frac{d \Phi\left(E_{v}\right)}{d E_{v}}=1.2 \cdot 10^{-8} e^{-E / 3 \mathrm{PeV}} \mathrm{GeV} \mathrm{cm}^{-2} \mathrm{~s}^{-1} \mathrm{sr}^{-1}
$$

even though different fits are also possible. The results presented here do not depend strongly on the assumed signal flux.

\section{Searches for diffuse neutrino fluxes}

Two analyses have been developed to search for a diffuse neutrino flux with ARCA. The "track" analysis searches for through-going muon track events induced mainly by $v_{\mu} \mathrm{CC}$ interactions and the shower analysis that identifies all-flavour neutrino interactions which produce electromagnetic and hadronic showers at the interaction vertex. Both analyses have been optimised using the "model discovery potential" (MDP) [6] method for a $5 \sigma$ discovery of the spectrum given in Eq. (1).

\subsection{Track analysis}

The first step in the event selection for the track channel analysis is a cut on the reconstructed track direction to get rid of most of the atmospheric muons. After optimisation a cut to keep events with reconstructed zenith angle $\theta_{z e n}>80^{\circ}$ was chosen. This cut does not reject all the atmospheric muons: a significant fraction of down-going atmospheric muons is wrongly reconstructed as up-going. To reject the background due to mis-reconstructed atmospheric muons and to atmospheric neutrinos the MDP minimisation is performed selecting well-reconstructed tracks and removing less energetic events mainly due to atmospheric neutrinos. The two reconstruction parameters correlated with the reconstruction quality and the neutrino energy are the $\Lambda$ and $N_{\text {hit }}$ parameters, described in [7]. After one year of operation with the ARCA detector, a flux twice that of Eq. (1) can be discovered with $5 \sigma$ significance. The sensitivity can be increased by using a statistical method based on the maximization of a likelihood function, rather than using a simple cut. This has been performed as described in [8], on a sample of preselected neutrino events with $\theta_{z e n}>80^{\circ}$ and a $\Lambda$ selection. The likelihood ratio function that has been maximised is given by:

$$
L R=\sum_{i=1}^{n} \log \frac{\frac{n_{s}}{n} P_{S}\left(N_{h i t}^{i}\right)+\left(1-\frac{n_{s}}{n} P_{b}\left(N_{h i t}^{i}\right)\right)}{P_{b}\left(N_{h i t}^{i}\right)}
$$

where $n_{s}$ is the number of signal events, $n_{b}$ the number of background events, $n=n_{s}+n_{b}$ and $P_{s}$ and $P_{b}$ are the probability distribution functions (PDF) for a signal or background to be reconstructed with a final selected number of hits $N_{\text {hit }}^{i}$. This function is maximised with respect to the number of signal events $n_{s}$. The resulting significance is reported in Fig. 1 as a function of the number of observation years and reaches about $4 \sigma$ after one year. 

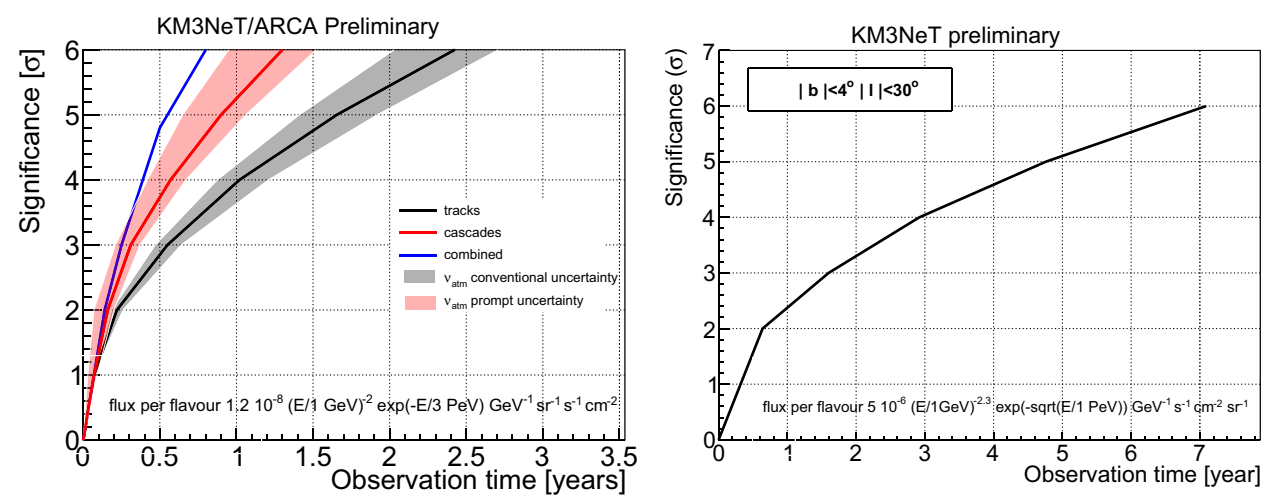

Figure 1. a) Discovery potential to the flux of Eq. (1) for ARCA analyses (see text for details). b) Performance of the track analysis in the search for a diffuse neutrino flux from the galactic plane.

\subsection{Showers}

An event selection procedure based on three consecutive cuts has been used in the shower analysis to isolate signal from background events. At the first selection level, only contained events are kept. Since most of the atmospheric muons enter the detector from above, their pseudo-vertices are reconstructed in the upper part of the detector. A large part of the background is rejected by cutting on coordinates of the reconstructed vertices with respect to the centre of the apparatus. A further rejection of atmospheric tracks, both from atmospheric muons and neutrinos, via an energy-related cut is obtained cutting on the total time over threshold (ToT) of hits selected by the shower reconstruction algorithm. The ToT is measured for each PMT and is a measure of the collected charge. Finally the signal is isolated using a machine-learning algorithm based on the boosted decision tree (BDT) from the ROOT TMVA package [9]. Quality parameters from the available shower and track reconstruction algorithms are used for the BDT classification. Its output is used together with the reconstructed shower energy $E_{\text {rec }}$ to separate the signal from both the remaining atmospheric neutrino and muon backgrounds. In the shower channel a $5 \sigma$ discovery of the assumed IceCube flux of can be achieved after 1.3 years of ARCA. A further improvement of this result is reached by applying the maximum likelihood method described above. The resulting significance as a function of the observation years is reported in Fig. 1.

\subsection{Combined search}

As both the track and shower analyses have a strong bias in efficiency towards one particular event topology, a further analysis strategy has been developed to incorporate the results from both the searches. The two analyses are indeed almost independent, as the overlap between the final event samples is limited to less then $2 \%$ of the surviving events. In order to develop an all-sky analysis with tracks and showers, the two hemispheres are treated separately. For downward-going events, where the atmospheric background is extremely high, only contained shower events are considered. The selection is then analogous to that of the usual shower analysis. For upgoing events, preliminary cuts are used to reject atmospheric muons that are wrongly reconstructed as upgoing, then a BDT method is used for a further rejection of background events. The BDT uses parameters coming from both the track and shower reconstruction algorithms and is optimised to reject atmospheric muons. Its output is used together with the shower energy estimation in the same maximum likelihood approach reported before. As a result a $4.8 \sigma$ significance is obtained in 0.5 years of observation time, as shown in Fig. 1. 


\section{Diffuse neutrinos from the Galactic plane}

One of the most promising regions of the sky from which a neutrino flux is expected is the Galactic Plane (GP) region. Neutrinos are expected to be produced in the interactions of cosmic rays with the dense galactic interstellar medium and radiation fields. The observation of $\gamma$-rays from the GP [10], part of which is expected to arise from the same hadronic processes that would produce high-energy neutrinos, strongly supports this hypothesis. Aiming at explaining the observed IceCube neutrino signal, some models evaluating the neutrino flux from regions in the GP near the Galactic center have been proposed. In [11] a CR propagation model with non uniform local transport properties has been adopted to explain the high energy $\gamma$-ray observation. To estimate the performance of the ARCA detector in searching for neutrinos from the GP, the resulting neutrino flux estimation has been adopted. In this framework the neutrino flux from a limited region of the GP near the galactic centre $\left(|l|<30^{\circ}\right.$ and $\left.|b|<4^{\circ}\right)$, with $b$ and $l$ being the galactic latitude and longitude respectively, has been estimated and the performances of the ARCA detector have been evaluated following the same approach as in Sect. 2.1. The result of the maximum likelihood approach for the track channel is shown in Fig. 1b. After about 5 years of operation the ARCA detector will be able to discover such a signal at a significance level of $5 \sigma$. As a consequence its contribution to the IceCube diffuse flux would be estimated and constraints on CR propagation models in the inner part of our Galaxy could be placed.

\section{Conclusions and outlook}

Thanks to the purity of the event sample, a high-significance detection of a cosmic neutrino signal will be possible for both track-like and cascade-like events within one year of operation. The excellent angular and energy resolutions combined with the large effective mass, provide for a significant discovery potential to find neutrino sources in the Universe. A diffuse component from the Galactic plane could be identified in a few years of operation, bringing fundamental knowledge about the physics of primary cosmic rays in the Milky Way.

\section{References}

[1] S. Adrián Martínez et al., KM3NeT 2.0 Letter of Intent, arXiv : 1601.07459 (2016)

[2] C. Kopper et al., proceedings of the $34^{\text {th }}$ ICRC, ID 1081, The Hague, The Netherlands (2015)

[3] M.G. Aartsen et al., Phys. Rev. D 91, 022001 (2015)

[4] M.G. Aartsen et al., Astrophys. J. 809, 98 (2015)

[5] M.G. Aartsen et al., Phys. Rev. Lett. 114, 171102 (2015)

[6] S. Adrián Martínez et al., Astr. Phys. 42, 7-14 (2013)

[7] A. Trovato et al., proceedings of the $34^{\text {th }}$ ICRC, ID 1279, The Hague, The Netherlands (2015)

[8] A. Trovato et al., proceedings of the $34^{\text {th }}$ ICRC, ID 1282, The Hague, The Netherlands (2015)

[9] A. Hoecker et al., TMVA - Toolkit for Multivariate Data Analysis, PoS ACAT 040 (2007)

[10] M. Ackermann et al., Astrophys. J. 750, 3 (2012)

[11] D. Gaggero et al., arXiv:1508.03681 (2015) 